\title{
Leiharbeit in Europa: zwischen Flexibilität und Sicherheit
}

\author{
Beschleunigt durch die Umsetzung der EU-Leiharbeitsrichtlinie und Kampagnen der Ge- \\ werkschaften steht die Regulierung der Leiharbeit in Deutschland derzeit (wieder) im \\ Mittelpunkt beschäftigungspolitischer Debatten. Die aktuell diskutierten Lösungsansätze \\ konzentrieren sich vor allem auf das Problem, die mit Leiharbeit verbundenen Einkom- \\ mensnachteile zu beseitigen und Lohngerechtigkeit (Equal Pay) zu erreichen. Andere \\ Regelungsbedarfe wie die fehlende Beschäftigungssicherheit in Leiharbeit oder die not- \\ wendige Stabilisierung der beruflichen Perspektiven von Leiharbeitnehmern bleiben weit- \\ gehend außer Acht. Gerechtigkeit und Chancengleichheit auf dem Arbeitsmarkt sollten \\ jedoch mehr beinhalten als bloße Einkommensaspekte. Hier kann Deutschland von an- \\ deren europäischen Ländern lernen, in denen weitergehende Regulierungsansätze ent- \\ wickelt worden sind.
}

ECKHARD VOSS, KATRIN VITOLS

\section{Einleitung}

Die massive Ausweitung der Leiharbeit und anderer Formen atypischer Beschäftigung infolge der Hartz-Reformen nach 2003 hat dazu beigetragen, dass Deutschland heute zwar im arbeitsmarktpolitischen Vergleich der Europäischen Union (EU) gut dasteht - am 1. März 2013 lag die Arbeitslosenquote laut Eurostat mit 5,3 \% nur halb so hoch wie in der EU insgesamt (10,8\%) -, gleichzeitig jedoch innerhalb der Europäischen Union und vor allem der Eurozone mit über $22 \%$ einen Spitzenwert beim Beschäftigungsanteil im Niedriglohnsektor belegt (vgl. Schulten 2013, S. 131).

Angesichts der derzeit diskutierten sozialen und ordnungspolitischen Korrekturmaßnahmen, die von der Abschaffung der Minijobs über Mindestlohnregelungen bis hin zu Branchenzuschlagsregelungen für Leiharbeitnehmer reichen, fällt auf, dass Erfahrungen anderer europäischer Länder in der Gestaltung und Regulierung der Leiharbeit bislang nur wenig berücksichtigt wurden (vgl. auch Vanselow/Weinkopf 2009), obgleich es seit 2008 eine europäische Rahmengesetzgebung gibt, die mittlerweile in allen EU-Ländern implementiert ist.

Die betrieblichen und arbeitsmarkpolitischen Funktionen der Leiharbeit weisen in Europa eine Reihe von Gemeinsamkeiten auf: Entsprechend der EU-Richtlinie gilt Leiharbeit zunächst als Instrument der Erhöhung (externer) betrieblicher Flexibilität im Sinne einer kurzfristigen und temporären Bereitstellung zusätzlicher Arbeitskräfte. Darüber hinaus legen neuere, auf die Leiharbeit in Deutschland bezogene Untersuchungen und Diskussionsbeiträge nahe, dass Leiharbeit zunehmend auch als strategisches Element des betrieblichen Personaleinsatzes genutzt wird, sei es als Screening-Instrument oder auch als Möglichkeit, Personalund Verwaltungskosten zu senken (Promberger 2012; Holst et al. 2009; Spermann 2011). Dieser Befund wird auch für andere europäische Länder bestätigt (Michon 2011, S. 126; ILO 2011; Belkacem et al. 2011).

In allen europäischen Ländern besteht eine Besonderheit der Leiharbeit darin, dass sie nicht nur als Mittel zur Erhöhung betrieblicher Flexibilität wahrgenommen wird, sondern dass ihr auch eine positive arbeitsmarktpolitische Funktion zugeschrieben wird. So betont etwa die EU-Richtlinie, dass Leiharbeit den Arbeitsmarktzugang insbesondere für solche Bevölkerungsgruppen erleichtern könne, die aufgrund geringerer Bildungs- und Qualifikationsstandards, längerer Arbeitslosigkeit oder sonstiger Eigenschaften (z. B. Alter, Migrationshintergrund) benachteiligt sind; zudem erwartet man sich von der Leiharbeit, dass sie als Brücke in reguläre Beschäftigungsverhältnisse funktioniere. In der Tat ist für alle europäischen Länder festzustellen, dass Leiharbeitnehmer im Durchschnitt jünger sind als die Beschäftigten insgesamt, dass ihr Bildungsniveau geringer ist und benachteiligte Gruppen, wie beispielsweise Personen mit Migrationshintergrund, ältere Arbeitnehmer oder vormals 
Langzeitarbeitslose in dieser Beschäftigungsform überrepräsentiert sind.

Wenngleich diese „doppelte“ Brückenfunktion der Leiharbeit in vielen Ländern der EU (insbesondere in Süd- und Osteuropa) als die wesentliche Legitimation dafür diente, bestehende Restriktionen der Leiharbeit aufzuheben bzw. zu lockern, diesen Beschäftigungssektor also zu deregulieren und auszubauen, ist nach wie vor die empirische Evidenz für diese Annahme umstritten. Die relativ umfangreiche Forschung hat bislang weniger eine nennenswerte Brückenfunktion bestätigt als vielmehr das Bild eines „schmalen Steges“ geprägt, wenn es um Übergänge aus der Zeitarbeit in Normalarbeitsverhältnisse geht. ${ }^{2}$

Keineswegs kann jedoch davon ausgegangen werden, dass Leiharbeit ein quasi automatisches Sprungbrett in Normalarbeitsverhältnisse darstellt. Um diese Funktion zu stärken, bedarf es, so die hier vertretene These, einer aktiven Gestaltung der Leiharbeit und Unterstützung der Leiharbeitnehmer im Sinne des Ansatzes sozial abgesicherter Übergangsarbeitsmärkte; es geht also darum, über Regelungen zur Einkommensgerechtigkeit hinauszugehen.

Im Mittelpunkt des folgenden Beitrags ${ }^{3}$ stehen daher Erfahrungen in der Regulierung und Gestaltung von Leiharbeit in anderen europäischen Ländern und insbesondere die Frage, welche Ansätze seitens der Sozialpartner in der Leiharbeitsbranche in Ergänzung zu gesetzlichen Regulierungsmechanismen entwickelt wurden, um die Lebens- und Arbeitsverhältnisse von Leiharbeitnehmern zu verbessern und Übergangschancen in Normalarbeitsverhältnisse zu vergrößern, um dadurch zu verhindern, dass Leiharbeit für die Beschäftigten zur „Niedriglohnfalle“ wird.

Abschnitt 2 vermittelt einen kurzen Überblick der grundlegenden Regulierungsansätze der Leiharbeit in der EU und weist auf die Bedeutung der tarifpolitischen Regelungen für die betriebliche und arbeitsmarktpolitische Funktion der Leiharbeit hin (2.1). Mit Blick auf die Inhalte konkreter Vereinbarungen in ausgewählten Ländern (2.2 und 2.3) wird deutlich, dass es Beispiele gibt, die über die Thematisierung der Einkommensungerechtigkeit hinausgehen und insbesondere Aspekte der Förderung der Weiterbildung und der sozialen Sicherung in den Fokus genommen haben. Wenngleich es an verlässlichen und insbesondere vergleichbaren Daten mangelt, wird kurz der verfügbare Kenntnisstand über positive Effekte der beschriebenen Maßnahmen zusammengefasst (2.4), bevor im Rahmen eines Fazits Schlussfolgerungen mit Blick auf die Diskussion über Leiharbeit in Deutschland abgeleitet werden (3).

\section{Leiharbeit als Gegenstand der Tarif- beziehungen}

\subsection{Die Bedeutung tarifpolitischer Regulierung}

Hinsichtlich der Regulierung von Leiharbeit in der EU ist zunächst festzustellen, dass diese in den meisten EU-Ländern jüngeren Datums ist: Während Länder wie Belgien, die Niederlande, Frankreich, Deutschland oder das Vereinigte Königreich bereits vor Jahrzehnten eine gesetzliche Legitimationsgrundlage geschaffen haben, wurde Leiharbeit in 15 der mittlerweile 28 EU-Länder erst im letzten Jahrzehnt rechtlich sanktioniert, oftmals als Folge der EU-Richtlinien-Politik.

Neben den Unterschieden im Reifegrad der gesetzlichen Regulierung bestehen substanzielle länderspezifische Differenzen in der gesetzlichen Regulierung von Leiharbeit in der betrieblichen Praxis: Während diese Form der Beschäftigung in einigen Ländern keinen Sonderstatus gegenüber dem Normalarbeitsverhältnis einnimmt (so z. B. im Vereinigten Königreich, in Irland, den skandinavischen
1 Gleichzeitig gibt es länderspezifische Besonderheiten, etwa signifikante Anteile von 30-40\% Hochqualifizierter in Leiharbeit in Ländern wie Bulgarien, Belgien, Schweden oder dem Vereinigten Königreich. Auffällig ist auch, dass in Ländern wie Frankreich, Belgien oder den Niederlanden eine relativ starke Minderheit von Leiharbeitnehmern (10-20\%) diese Form der Beschäftigung nutzt, um ein Studium zu finanzieren oder einen Hinzuverdienst zur Haus- und Familienarbeit oder Rente zu erzielen. Hier erfüllt die Leiharbeit eine arbeitsmarktpolitische Funktion, die in Deutschland eher durch geringfügige Beschäftigung besetzt ist.

2 Vgl. entsprechende Befunde zu Deutschland: Lehmer/Ziegler 2010; Crimmann et al. 2009; zu Frankreich: Observatoire des Métiers et de I'Emploi 2011; zu den Niederlanden: Dekker et al. 2011 und zu Belgien: IDEA Consult 2009.

3 Dieser Beitrag basiert auf den Ergebnissen einer europäischen Studie, die von den Autoren gemeinsam mit Kooperationspartnern in Belgien, Frankreich, Italien und dem
Vereinigten Königreich im Jahre 2012 im Auftrag der europäischen Sozialpartner in der Leiharbeitsbranche, UNI Europa und Eurociett, durchgeführt worden ist. Ziel der Studie "Temporary Agency Work and Transitions in the Labour Market" war es, einen aktuellen Überblick über Systeme, Funktionen und Profile der Leiharbeit in Europa zu leisten und insbesondere die Rolle des sozialen Dialogs und der Sozialpartner bei der Ausgestaltung von Rahmenbedingungen zu bewerten (zu den Ergebnissen vgl. Voss et al. 2013). Im Rahmen der Studie wurden ferner erstmals national verfügbare empirische Daten und Forschungsergebnisse zur Rolle der Leiharbeit für verschiedene Formen von Arbeitsmarktübergängen und zur Brückenfunktion von Leiharbeit aufgearbeitet und verglichen. Ergebnisse der Studie wurden im Rahmen des gemeinsamen Sozialdialog-Komitees der europäischen Sozialpartner und der EU-Kommission sowie einer internationalen Konferenz im Dezember 2012 präsentiert und bildeten die Grundlage einer gemeinsamen Erklärung von UNI-Europa und Eurociett zu Leiharbeit und Arbeitsmarktübergängen. 
ABB. 1

\section{Regulierungsmuster der Leiharbeit in der EU-27}

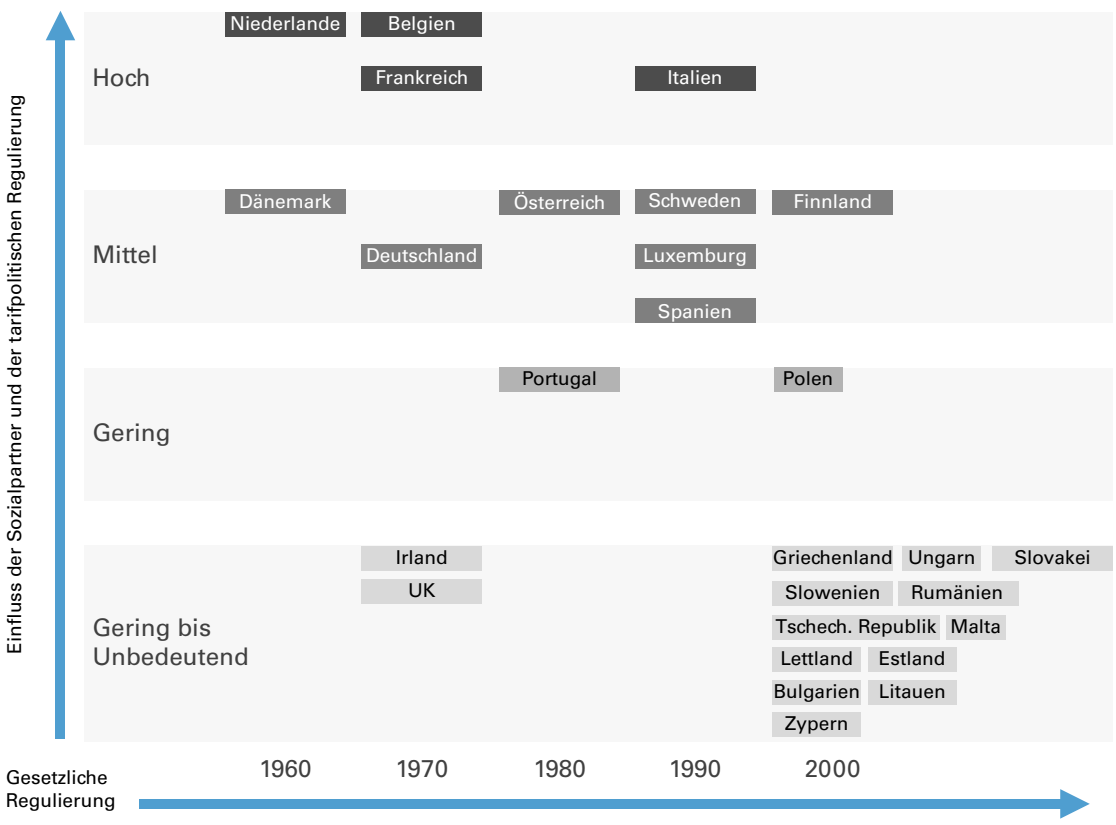

Quelle: Voss et al. (2013, S. 10); Darstellung der Autoren.

Ländern), ist sie in anderen Ländern durch eine Vielzahl von leiharbeitsspezifischen Sonderregelungen beeinflusst. ${ }^{4}$

In welchem Maße Leiharbeit Gegenstand tarifpolitischer Regulierung und aktiver Einflussnahme der Sozialpartner (bzw. tripartistischer Regulierung) ist, hängt stark von den jeweiligen Modellen und Systemen der industriellen Beziehungen und der Tarifpolitik ab: Lediglich in elf Ländern ${ }^{5}$ spielen Tarifverhandlungen auf überbetrieblicher Ebene eine wichtige Rolle bei der Regulierung von Leiharbeit. Sehr unterschiedlich gehen dabei insbesondere die Gewerkschaften mit der Besonderheit des dreiseitigen Arbeitsverhältnisses in der Leiharbeit um: Während lediglich in Frankreich und Italien eigene Branchengewerkschaften bestehen, finden in allen Ländern Tarifverhandlungen nicht nur für die Leiharbeitsbranche selbst statt, sondern auch auf der Ebene der wichtigsten Entleihbranchen. In Belgien und Frankreich ist darüber hinaus die Leiharbeit auch Gegenstand des dreiseitigen Dialogs der Sozialpartner mit der Regierung, und tripartite Vereinbarungen spielen eine wichtige Rolle bei der Regulierung.

Was die Bedeutung der Sozialpartner und der Tarifverhandlungen für die Regulierung von Leiharbeit und der Arbeitsbedingungen in Leiharbeit angeht, so heben sich vier Länder deutlich vom Rest der EU ab, wie Abbildung 1 verdeutlicht: In den Niederlanden, Belgien, Frankreich und Italien spielen Gewerkschaften wie Arbeitgeber und deren Vereinbarungen eine eminent wichtige Rolle für die Regu-

lierung und konkreten Arbeitsbedingungen in der Leiharbeit. Zudem wurden hier spezifische Ansätze entwickelt, um Flexibilität und Beschäftigungssicherheit auszubalancieren sowie sonstige Schutzmechanismen zu etablieren, die in den folgenden Abschnitten näher beschrieben werden. Demgegenüber ist die Praxis in den übrigen EU-Ländern deutlich stärker von der jeweiligen Macht- und Einflussposition der Gewerkschaften geprägt. Tarifpolitische Vereinbarungen können hier - wie im Vereinigten Königreich und in den mittel- und osteuropäischen Ländern - bestenfalls für die betriebliche Ebene erzielt werden. Auch die jüngst in einzelnen Wirtschaftsbranchen erzielten Vereinbarungen über Branchenzuschläge in Deutschland (siehe Schwitzer/ Schumann 2013) und die teilweise beträchtlichen Unterschiede in den Zuschlagsniveaus einzelner Wirtschaftszweige verdeutlichen den Zusammenhang zur jeweiligen Organisationsstärke.

\subsection{Equal Pay und Gleichbehandlung von Leih- arbeitnehmern}

Die Umsetzung des Equal-Pay-Prinzips in der Leiharbeitsbranche in der EU ist geprägt von einer großen Heterogenität gesetzlicher Regelungen, die oftmals durch tarifvertragliche Vereinbarungen präzisiert oder ergänzt werden (vgl. Eurofound 2009, S. 41f.).

In Belgien gilt Equal Pay bereits seit 1976 uneingeschränkt, und die intensive und langjährige Zusammenarbeit zwischen den belgischen Gewerkschaftsbünden ABVV/ FGTB, ACLVB/CGSLB und ACV/CSC und der Arbeitgebervereinigung in der Leiharbeit, Federgon, hat dazu geführt, dass Leiharbeitnehmer auch ein Recht auf die gleichen sozialen Leistungen und Angebote wie Festangestellte im Entleihbetrieb haben, z. B. im Hinblick auf Essensgutscheine, Reisekostenpauschalen, finanzielle Beteiligungsprogramme, betriebliche Altersvorsorge etc.

Auch in Frankreich gilt seit der erstmaligen gesetzlichen Regulierung 1972 das Prinzip „Equal Pay“ vom ersten Tag an, und es existiert darüber hinaus ein sogenannter „Prekaritäts-Bonus“ in Höhe von 10 \% des Entgelts, der Leiharbeitnehmer nicht nur für die erhöhte Arbeitsplatzunsicherheit, sondern auch dafür entschädigen soll, dass sie z. B. nicht an der in vielen Unternehmen vorgeschriebenen Ge-

4 Solche Sonderregelungen gibt es bspw. hinsichtlich des arbeitsvertraglichen Verhältnisses, der erlaubten Gründe, Leiharbeit einzusetzen, Befristungen, höchstzulässiger Überlassungsdauer, maximaler Anzahl der Einsätze bei einem Entleiher, Verbot der Arbeitnehmerüberlassung für bestimmte Tätigkeiten, Berufe oder Branchen und nicht zuletzt der Umsetzung des in der EU-Richtlinie formulierten Lohngleichheitsprinzips und der Gleichstellung (vgl. Voss et al. 2013, S. 28ff.)

5 Dies sind: Österreich, Belgien, Dänemark, Finnland, Frank reich, Deutschland, Italien, Luxemburg, Niederlande, Spanien, Schweden. 
winnbeteiligung partizipieren. Darüber hinaus haben Vereinbarungen zwischen den Gewerkschaftsorganisationen CFDT, CFE-CGC-FNECS, CFTC, USI-CGT, CGT-FO und dem Arbeitgeberverband PRISME einen wichtigen Beitrag zur Regulierung der Arbeitsbedingungen geleistet, die im Folgenden (2.3) näher erläutert wird.

In der italienischen Gesetzgebung ist ebenfalls der Grundsatz der Gleichbehandlung von Leiharbeitnehmern mit Arbeitnehmern im Einsatzunternehmen hinsichtlich Entgelt, Urlaub, Arbeitszeitregelungen, Pausen oder Mutterschutz fest verankert und wurde im Zuge einer Arbeitsmarktreform im Juli 2012 noch einmal verstärkt. Im Zuge der sogenannten „Fornero Reform“ im Sommer 2012 wurde eine Klausel aus dem Jahr 2003 abgeschafft, die eine Abweichung vom Equal-Pay-Prinzip dann zuließ, wenn benachteiligte Beschäftigte durch Leiharbeit eingestellt wurden. Heute gilt Equal Pay dagegen uneingeschränkt. Seit der erstmaligen Legalisierung der Arbeitnehmerüberlassung 1997 durch den Gesetzgeber spielen in Italien die Sozialpartner in der Ausgestaltung der Leiharbeit eine besondere Rolle, da hier die branchenweiten Tarifvereinbarungen zwischen den drei Gewerkschaftsorganisationen der Leiharbeitsbranche NIDiL, FELSA, UILTemp sowie der Arbeitgeberorganisation Assolavoro eine rechtliche Bindewirkung entfalten.

Das niederländische Regulierungsmodell der Leiharbeit und insbesondere die Regelungen zu Equal Pay sind aus deutscher Sicht interessant: So ist Leiharbeit in den Niederlanden ähnlich stark dereguliert wie in Deutschland, und es gibt keinerlei Beschränkungen in einzelnen Branchen oder hinsichtlich der betrieblichen Gründe und Einsatzdauer. Weiterhin basiert auch das niederländische Modell der Leiharbeit auf dem „Arbeitgeberprinzip“, d.h. Leiharbeitsagenturen gelten als normale Arbeitgeber und haben alle arbeitsrechtlichen Verpflichtungen gegenüber den Beschäftigten. Mit Blick auf die Umsetzung des Equal-PayGrundsatzes hat die niederländische Gesetzgebung schließlich einen ähnlichen Weg wie Deutschland gewählt und die konkrete Umsetzung den Tarifpartnern und tariflichen Vereinbarungen überlassen, einschließlich der Möglichkeit, vom Grundsatz der Lohngleichheit abzuweichen.

Bereits mit dem Branchenvertrag im Jahre 1999 wurde dabei ein System geschaffen, in dem Leiharbeitnehmer bestimmte Phasen durchlaufen, die mit jeweils weitergehenden Ansprüchen (z. B. Lohnfortzahlung in einsatzfreien Zeiten) verbunden sind. Das Modell basiert derzeit auf drei unterschiedlichen Phasen, die einen Zeitraum von dreieinhalb Jahren umfassen. Hinsichtlich des Equal-Pay-Prinzips beinhaltet die Tarifvereinbarung der Leiharbeitsbranche, dass sich bis 2015 das Entgelt eines Leiharbeitnehmers innerhalb der ersten 26 Wochen des Einsatzes entweder am Tarifvertrag der Leiharbeitsbranche orientiert oder an der Lohnhöhe eines vergleichbaren Arbeitnehmers im Einsatzunternehmen. Ab 2015 gilt dann das Prinzip der Lohngleichheit (gleicher Lohn für gleiche Arbeit) uneingeschränkt von Beginn an.

\subsection{Vereinbarungen zur sozialen Sicherung und Verbesserung der Beschäftigungsperspektiven}

Ein zentrales Instrument, um die Beschäftigungsperspektiven von Leiharbeitnehmern zu verbessern und Arbeitsmarktübergänge zu fördern, sind Weiterbildungsfonds und tarifliche Vereinbarungen zur Unterstützung der Übergänge in stabilere Beschäftigungsformen. Während in Deutschland die Arbeitgeberverbände in der Leiharbeit hier vor allem auf das Engagement der Leiharbeitsagenturen verweisen und branchenweite Lösungen ablehnen, existieren derartige Vereinbarungen in anderen EU-Ländern bereits seit Langem. Sie beschränken sich nicht nur auf die Weiterbildung, sondern gehen (wie etwa in Belgien, Frankreich, Italien, Spanien, den Niederlanden sowie neuerdings auch in Österreich $)^{6}$ darüber hinaus und beinhalten auch Aspekte der sozialen Sicherung und der Altersvorsorge.

\subsubsection{Belgien}

Seit 1993 regelt in Belgien eine gemeinsame Tarifkommission Fragen wie die Aus- und Weiterbildung von Leiharbeitnehmern, ihre Gleichstellung, die Förderung von Leiharbeitnehmern mit Migrationshintergrund oder Probleme der Altersvorsorge. Seit 2003 besteht ferner ein gemeinsamer Code of Good Practice, in dem wesentliche Grundsätze guter Entleihpraxis festgehalten sind. Im Jahre 2006 wurde der Weiterbildungsfonds (vfu-ffi) sowie der Sozialfonds FSI durch einen Tarifvertrag geschaffen, der sich aus Abgaben der Leiharbeitgeber (0,4 \% der Lohnsumme) finanziert und gemeinsam durch die Sozialpartner verwaltet wird. Im Zuge der Umsetzung der EU-Leiharbeitsrichtlinie wurde 2012 in einer Vereinbarung der Sozialpartner und der belgischen Regierung ferner eine Reihe von Maßnahmen verabschiedet, die einerseits darauf abzielen, das Instrument Leiharbeit stärker für arbeitsmarktpolitische Zielsetzungen nutzbar zu machen (Lockerung bzw. Erweiterung der betrieblichen Einsatzgründe und Einführung eines Eingliederungsvertrages für vormals Arbeitslose, Lockerung zeitlicher Beschränkungen), die andererseits aber auch die Schutzregelungen verstärken. So sind Arbeitgeber nunmehr z. B. verpflichtet, Leiharbeitnehmer auf freie Stellen im Unternehmen hinzuweisen.

6 In Österreich wurde im September 2012 eine Novelle zum Arbeitskräfteüberlassungsgesetz (AÜG) beschlossen, mit der einerseits auf die Vorgaben der EU-Richtlinie zur Gleichbehandlung reagiert wurde und andererseits weitergehende Maßnahmen zur Verbesserung der Arbeitsmarktsituation von Leiharbeitnehmern zwischen den Sozialpartnern eingeleitet wurden. Dazu gehört insbesondere die Einrichtung eines Sozial- und Weiterbildungsfonds, der Anfang 2014 an den Start gehen wird. 


\subsubsection{Frankreich}

Bereits im Jahre 1983 wurden in Frankreich zwei Weiterbildungsfonds, der Fonds d'assurance formation du travail temporaire (FAF.TT) und der Fonds Professionnel pour l'Emploi dans le travail temporaire (FPE.TT) geschaffen, die sich im Wesentlichen aus Beiträgen der Arbeitgeber finanzieren (insgesamt 2,15\% der Lohnsumme) und ebenfalls in gemeinsamer Regie der Arbeitgeber und Gewerkschaften verwaltet werden. Dabei soll der FAF.TT die Leiharbeitsagenturen vor allem bei der Integration und Qualifizierung von vormals arbeitslosen Beschäftigten unterstützen. Außer den Aktivitäten der Fonds haben die französischen Sozialpartner in Branchenvereinbarungen weitere Programme der beruflichen Bildung und Weiterbildung aufgelegt, etwa 2004 die CIPI (Contrat d'insertion professionnelle intérimaire) und CDPI (Contrat de développement professionnel intérimaire), mit denen insbesondere die berufliche Entwicklung von benachteiligten Personengruppen gefördert werden soll. Auch im Bereich der beruflichen Erstausbildung haben die Sozialpartner in der Leiharbeitsbranche Instrumente geschaffen (Contrat de professionnalisation und die Période de professionnalisation), um vor allem jüngeren Menschen ohne Berufsabschluss grundlegende berufliche Qualifikationen zu vermitteln und damit den Einstieg in den Arbeitsmarkt zu fördern.

Schließlich organsiert der Sozialfonds FAS.TT (Fonds d'action sociale du travail temporaire), der ebenfalls gemeinsam von den Sozialpartnern getragen wird, unterschiedliche Formen sozialer Unterstützung für Leiharbeitnehmer, die von Hilfen bei der Tagespflege für Kinder bis hin zu Mobilitätshilfen und günstigen Darlehen oder Bürgschaften bei Umzug oder Führerscheinerwerb reichen. Eine Untersuchung des FAS.TT aus dem Jahre 2007 illustriert die Bedeutung solcher Einrichtungen: 55 \% der Leiharbeitnehmer, die einen Kredit bei dem Fonds aufgenommen haben, hatten dies zuvor erfolglos bei einer Bank versucht.

\subsubsection{Italien}

Auch in Italien wurden bilaterale Fonds und Institutionen etabliert, so etwa 2002 der Weiterbildungsfonds Forma. Temp oder 2008 der Sozialfonds Ebi.Temp und die Pensionskasse Fon.Temp - allesamt paritätische Einrichtungen, die entweder aus Arbeitgeberabgaben oder freiwilligen Beiträgen der Arbeitnehmer (Pensionskasse) finanziert werden. ${ }^{7}$ Ähnlich wie in Frankreich oder Belgien ist dabei der Aufgabenzuschnitt des Sozialfonds recht breit und reicht von zusätzlichen Unterstützungen im Krankheitsfall bis hin zu günstigen Darlehen.

Die nationalen Tarifabkommen in der Leiharbeitsbranche in Italien beinhalten auch Maßnahmen zur Förderung von Arbeitsmarktübergängen, wie z. B. Informationspflichten der Leiharbeitgeber gegenüber den territorialen Gewerkschaftsorganisationen, zur maximalen Verweildauer bzw. Verlängerung von Einsatzverträgen oder zu sozialen
Leistungen wie Mutterschutz, zusätzlichen Krankengeldleistungen, Zuschüssen zur Krankenversicherung oder Einmalzahlungen bei Entlassungen. Erklärtes Ziel der Tarifvereinbarung ist die Förderung von Übergängen in direkte und unbefristete Beschäftigung. Leiharbeitnehmer haben in der Regel ab einer Einsatzdauer von 36 Monaten das Recht auf eine direkte und unbefristete Festanstellung.

\subsubsection{Die Niederlande}

Auch in den Niederlanden war es das Ziel des Gesetzgebers und der Sozialpartner, die mit Leiharbeit (und anderen Formen flexibler Beschäftigung) verbundene größere Arbeitsplatzunsicherheit durch gezielte Maßnahmen und Instrumente des Beschäftigungsschutzes und der Beschäftigungsfähigkeit zu flankieren, die über Aspekte der Einkommensgerechtigkeit hinausgehen (Houwing 2010).

Angesichts der deutlichen Zunahme flexibler Beschäftigungsformen und der Forderungen der Arbeitgeberorganisationen ABU und NBBU nach Deregulierung sowie dem Bemühen der Gewerkschaftsverbände FNV, CNV und De Unie, stärkere Schutzmechanismen zu verankern, wurden Ende der 1990er Jahre zwei gesetzliche Regelungen getroffen, welche die Leiharbeit in den Niederlanden grundlegend reformierten: Während 1998 das Leiharbeitsgesetz WAADI (Wet Allocatie Arbeidskrachten Door Intermediairs) eine weitgehende Deregulierung der Leiharbeit bewirkte (u.a. Abschaffung der Lizenzierung und bestehender Beschränkungen der Leiharbeit), zielt das 1999 in Kraft getretene Gesetz zu Flexibilität und Sicherheit flexwet (Wet Flexibiliteit en Zekerheid) darauf ab, die arbeitsmarktpolitische Situation von Leiharbeitnehmern sowie deren soziale Absicherung zu verbessern und Maßnahmen zu ergreifen, die sich positiv auf Übergänge in Normalarbeitsverhältnisse auswirken. Auf dieser gesetzlichen Basis sind seit 1999 Tarifabkommen zwischen den Sozialpartnern in der Leiharbeitsbranche abgeschlossen worden, in denen wesentliche Aspekte der Arbeitsbedingungen und der Förderung von Übergängen in unbefristete Beschäftigung enthalten sind. Dies beinhaltete auch die Schaffung von bilateralen Institutionen im Bereich der Ausund Weiterbildung (stoof), des Arbeits- und Gesundheitsschutzes (staf) sowie der Überwachung tariflicher und arbeitsrechtlicher Regelungen (sncu), die unter dem Dach eines Sozialfonds (sfu) organisiert sind. Die Arbeit der Fonds wird durch eine jährliche Abgabe der Leiharbeitsfirmen in Höhe von 0,2 \% der Lohnsumme finanziert.

Die „Stiftung für Training und Entwicklung“ (stoof) wurde 2003 von den niederländischen Sozialpartnern als zentrale Einrichtung für Weiterbildungsaktivitäten gegrün-

7 Durch die Arbeitsmarktreform im Juli 2012 wurde der Finanzierungsbeitrag neu geregelt: Zukünftig beträgt der Arbeitgeberanteil nur noch 2,6\% der Lohnsumme, während weitere 1,4\% aus den Kassen der Sozialversicherung INPS beigesteuert werden. 
det und leistet finanzielle Unterstützung, zertifiziert Bildungsmaßnahmen, entwickelt Curricula und fördert die Zusammenarbeit mit anderen Branchen und Akteuren der Weiterbildung. Im Branchentarifvertrag 2009 wurde ferner vereinbart, besondere Anstrengungen für Geringqualifizierte zu erbringen und ein regelmäßiges Monitoring von Weiterbildungsaktivitäten und -ergebnissen durchzuführen (Vermeulen/Warmerdam 2011).

\subsection{Positive Effekte der Weiterbildungsinitiativen}

Wenngleich ländervergleichende Untersuchungen zur Weiterbildungsbeteiligung oder Qualifizierung von Leiharbeitnehmern bislang nicht existieren, so lassen sich doch empirische Belege dafür finden, dass die geschilderten Maßnahmen positive Effekte für die Beschäftigten und deren berufliche Perspektiven haben. Während Studien für Deutschland aufzeigen, dass Weiterbildung für Leiharbeitnehmer eher selten und wenig umfassend ist (Bellmann et al. 2013), kommen Untersuchungen für das europäische Ausland zu anderen Ergebnissen: So zeigen Untersuchungen in den Niederlanden, dass Weiterbildung und Qualifizierungsmaßnahmen heute in den meisten Leiharbeitsagenturen ein fester Bestandteil geworden sind, nicht nur in den größeren Unternehmen der Branche, sondern dank der Arbeit des Weiterbildungsfonds gerade auch in kleineren Agenturen (Vermeulen/Warmerdam 2011). Laut Angaben des Arbeitgeberverbandes ABU liegt die Weiterbildungsquote bei Leiharbeitnehmern zudem deutlich über derjenigen von Beschäftigten mit Teilzeitverträgen. Jüngere Studien verdeutlichen außerdem, dass Leiharbeitnehmer in den Niederlanden bessere Chancen auf eine reguläre Festanstellung haben als Arbeitslose und andere atypisch Beschäftigte (Vermeulen et al. 2012).

Eine hohe Beteiligung von Leiharbeitnehmern an Weiterbildungsmaßnahmen wird auch aus Frankreich berichtet (Möbus 2007), wenngleich es auch kritischere Analysen gibt (Erhel et al. 2009). Laut Untersuchungen des bilateralen Weiterbildungsfonds FAF.TT haben die Weiterbildungsmaßnahmen zu einer Verbesserung der Qualifikationsstandards beitragen können: Zwischen 2006 und 2011 ging der Anteil der Ungelernten an den Leiharbeitnehmern von 52 auf $23 \%$ zurück, während der Anteil der qualifizierten Beschäftigten sich von 22 auf $35 \%$ erhöht hat. Auch andere Studien bestätigen die positive Wirkung von beruflichen Qualifizierungsmaßnahmen auf den Status und die Karriereaussichten von Leiharbeitnehmern, insbesondere auch für jüngere Personen ohne oder mit geringen beruflichen Qualifikationen. Leiharbeitnehmer, die eine Trainingsmaßnahme absolvieren, haben deutlich bessere Chancen auf einen unbefristeten Anstellungsvertrag im Entleihunternehmen als diejenigen, die nicht an Trainingsmaßnahmen teilgenommen haben (FAF-TT 2012).

Schließlich bestätigen auch Angaben des Weiterbildungsfonds Ebi.Temp in Italien die positiven Effekte von Bildungsmaßnahmen. So haben Befragungen gezeigt, dass insbesondere für bestimmte Zielgruppen die Leiharbeit eine wichtige Möglichkeit darstellt, auf dem Arbeitsmarkt Fuß zu fassen. In starkem Kontrast etwa zu Untersuchungsergebnissen aus Deutschland (Siebenhüter 2011), die zu dem Ergebnis kommen, dass Leiharbeit aus Sicht von Migranten eher als Integrationshemmnis anzusehen ist, haben Befragungen in Italien ergeben, dass rund $30 \%$ der Migranten den Berufseinstieg über Leiharbeit wählen und dabei die Möglichkeiten zum Erlernen neuer Fähigkeiten sowie die Anerkennung in den Heimatländern erworbener Qualifikationen positiv hervorheben (Altieri et al. 2009).

\section{Fazit: Ein verlorenes Jahrzehnt für Deutschland}

Stärker als alle anderen EU-Länder ist Deutschland seit rund einem Jahrzehnt geprägt durch eine massive Zunahme von atypischen, oftmals prekären Arbeitsverhältnissen, die durch eine hohe Arbeitsplatzunsicherheit, geringe Löhne und entsprechend niedrige Sozialleistungen gekennzeichnet sind. Dies hat die Frage aufgeworfen, in welcher Weise die betroffenen Beschäftigten besser unterstützt werden können. Dabei wird neben der Forderung nach einem allgemeinen Mindestlohn oder nach Korrektur von eklatanten Fehlentwicklungen (wie im Bereich der Minijobs) immer wieder auch die besondere Bedeutung eines verbesserten Zugangs $\mathrm{zu}$ Weiterbildung hervorgehoben, der eine grundlegende Bedingung für Beschäftigungssicherheit darstellt (siehe Keller/Seifert 2011; Bellmann et al. 2013).

Mit Blick auf die Regulierung von Leiharbeit in anderen europäischen Ländern hat dieser Artikel deutlich gemacht, dass es konkrete Gestaltungsansätze in der Leiharbeit gibt, die nicht einzig auf Lohngerechtigkeit und Einkommenssicherheit zielen, sondern die auch auf eine Verbesserung der Beschäftigungsfähigkeit sowie die Förderung von Arbeitsmarktübergängen hinwirken.

Deutlich wird, dass Deutschland hier noch in den Kinderschuhen steckt. Allerdings konnten in jüngster Zeit zumindest eklatante tarifpolitische Fehlentwicklungen wie das Auftreten der CGZP-Tarifverträge korrigiert werden und mit den Vereinbarungen zu Branchenzuschlägen erste Ergebnisse in Richtung Einkommensverbesserung erzielt werden. Gleichzeitig verdeutlichen die hier beschriebenen Länderbeispiele aber auch, dass es weitergehender Gestaltungsansätze bedarf, um die Brückenfunktionen der Leiharbeit zu stärken und Beschäftigte bei Übergängen in Normalarbeitsverhältnisse aktiv zu unterstützen. Eine Schlüsselfunktion spielen hier die Erfahrungen der gemeinsamen Weiterbildungs- und Sozialfonds sowie entsprechende branchenweite Tarifvereinbarungen.

Hier ist der Blick auf die Rolle der Arbeitgeberorganisationen in Belgien, Frankreich und Italien höchst 
interessant, da es sich ja bei diesen Ländern keinesfalls um Fälle handelt, die für konfliktfreie und harmonische Beziehungen zwischen den Sozialpartnern bekannt sind. Gleichwohl verdeutlichen die hier geschilderten nationalen Beispiele, dass es zum Thema Leiharbeit Handlungsfelder gibt, in denen Arbeitgeber und Gewerkschaften ähnliche Interessen haben. $\mathrm{Zu}$ nennen ist hier insbesondere die Qualifizierung und Weiterbildung der Beschäftigten. Dabei haben die Arbeitgeberorganisationen nicht nur ein Interesse daran, das Image der Leiharbeitsbranche aufzuwerten, sondern auch daran, die Leiharbeit als beschäftigungspolitisches Instrument zur Förderung von Arbeitsmarktübergängen, insbesondere zur Integration von benachteiligten Gruppen in den Arbeitsmarkt, in der öffentlichen Diskussion und Wahrnehmung zu platzieren.

Wenngleich es nach wie vor insbesondere mit Blick auf konkrete Arbeitsbedingungen und die Qualität der Leiharbeit an vergleichenden und evidenzbasierten Studien und Untersuchungen fehlt (so mangelt es insbesondere an vergleichenden Daten und Analysen zur Einkommenssituation und zu den Effekten von Weiterbildungsaktivitäten), lassen doch die z. B. in den Niederlanden oder Frankreich vorhandenen Untersuchungen vermuten, dass sich die bilateralen Regulierungsansätze insbesondere auch im Bereich der beruflichen Bildung nicht nur für die Unternehmen - Leiharbeitgeber wie Nutzer - auszahlen, sondern auch positiv auf den Status und die Beschäftigungsperspektiven der in der Leiharbeit beschäftigten Menschen auswirken.

Verglichen mit den vorhandenen Ansätzen zur Regulierung der Leiharbeit in den hier beschriebenen Ländern fällt Deutschland zurück - zugespitzt ausgedrückt, kann man hierzulande von einem verlorenen Jahrzehnt (tarif)politischer Gestaltung sprechen. Das erstaunt umso mehr, als gerade angesichts des spezifischen deutschen Modells der industriellen Beziehungen auf betrieblicher wie branchenspezifischer Ebene zu erwarten gewesen wäre, dass sich allemal ähnliche Mechanismen der Regulierung und Ausbalancierung von Flexibilität und Sicherheit bzw. Förderung flexibler Arbeitnehmer herausbilden würden, wie dies in einigen anderen europäischen Ländern der Fall ist. Stattdessen aber ist der hiesige Umgang mit Leiharbeit nach wie vor geprägt von heftig geführten Debatten und Konflikten in betrieblichen, branchenpolitischen wie gesellschaftspolitischen Räumen. Es dominiert eine hartnäckige Verweigerungshaltung innerhalb des Arbeitgeberlagers, mehr Sicherheiten für Leiharbeitsbeschäftigte zu schaffen - es fehlt aber auch an einer branchenübergreifenden Strategie der Gewerkschaftsbewegung. Auch das Regierungshandeln lässt in puncto Leiharbeit eine arbeitspolitische Orientierung vermissen - zu prioritär ist das letztlich auch wahlpolitisch motivierte Ziel, mit einer möglichst geringen Arbeitslosenquote dazustehen, unabhängig davon, welche Nachteile die Leiharbeit für die Beschäftigten mit sich bringt. Insofern wurde die Debatte über die Regulierung von Leiharbeit in den letzten Jahren fast ausschließlich von der Kampagnenfähigkeit der Gewerkschaften in Gang gehalten bzw. durch die erforderliche Umsetzung des Gleichbehandlungsprinzips der EURichtlinie neu entfacht. Diese Impulse haben schließlich auch zu den Tarifvereinbarungen für Branchenzuschläge geführt.

Zu hoffen bleibt, dass sich die ordnungspolitischen Rahmenbedingungen zur Regulierung der Leiharbeit auch in Deutschland künftig weiter verbessern werden. Es geht um weitergehende tarifliche Vereinbarungen, etwa zur Verbesserung der sozialen Sicherung, zur Fort- und Weiterbildung von Leiharbeitnehmern (und anderen atypisch Beschäftigten); und es geht um Maßnahmen, die insgesamt geeignet sind, die Arbeitsmarktübergänge zu fördern und Segmentierungstendenzen entgegenzuwirken.

\section{LITERATUR}

Altieri, G./Dota, F./Piersanti, M./Angeli, F. (2009): Percorsi nel lavoro atipico. II caso dei lavoratori interinali. - L'indagine è stata condotta con tecnica C.A.T.I. Belkacem, R./Kornig, C./Michon, F. (2011): Visages de l'intérim en France et dans le monde, Paris

Bellmann, L./Grunau, P./Leber, U./Noack, M. (2013): Weiterbildung atypisch Beschäftigter: Bertelsmann-Stiftung, Gütersloh

Crimmann, A./Ziegler, K./Ellguth, P./Kohaut, S./Lehmer, F. (2009): Forschungsbericht zum Thema „Arbeitnehmerüberlassung", Endbericht: IAB, Nürnberg Dekker, R./Houwing, H./Kösters, L. (2011): Van flexibel naar vast? De doorstroom naar vast werk van een groeiende groep flexwerkers: CBS, Den Haag Erhel C./Lefevre G./Michon F. (2009): L'intérim: un secteur dual, entre protection et précarité, document de travail du Centre d'Économie de la Sorbonne, Université de Paris 1 - CNRS, 2009.14, Paris

European Foundation for the Improvement of Living and Working Conditions (Eurofound) (2009): Temporary Agency Work and Collective Bargaining in the EU, Dublin

Fond d'assurance formation du travail temporaire (FAF-TT) (2012): Formation et insertion des jeunes intérimaires, September

Holst, H./Nachtwey, O./Dörre, K. (2009): Funktionswandel von Leiharbeit. Neue Nutzungsstrategien und ihre arbeits- und mitbestimmungspolitischen Folgen, Studie im Auftrag der Otto Brenner Stiftung, Frankfurt a. M.

Houwing, H. (2010): A Dutch approach to Flexicurity? Negotiated change in the organization of temporary work, Academisch Proefschrift, Amsterdam IDEA Consult (2009): Temporary agency work: A springboard toward longterm employment, also for disadvantaged groups? Study commissioned by the Flemish Minister in charge of employment, education and training as part of the VIONA research programme, Juni, Brüssel

International Labour Organization (ILO) (2011): Private employment agencies, promotion of decent work and improving the functioning of labour markets in private services sectors: International Labour Office, Sectoral Activities Programme, Issues paper for discussion at the Global Dialogue Forum on the Role of Private Employment Agencies in Promoting Decent Work and Improving the Functioning of Labour Markets in Private Services Sectors, 18-19 October, Geneva

Keller, B./Seifert, H. (2011): Atypische Beschäftigung und soziale Risiken. Entwicklung, Strukturen, Regulierung, Bonn

Lehmer, F./Ziegler, K. (2010): Brückenfunktion der Leiharbeit - Zumindest ein schmaler Steg: IAB, IAB-Kurzbericht 13/2010, Nürnberg

Michon, F. (2011): Survey on non-regular employment in France. A profile, in: Japan Institute for Labour Policy and Training (JILPT): Non-regular employment. Issues and challenges common to the major developed countries, Seminar on Non-Regular Employment, JILPT (10), S. 117-140

Möbus, M. (2007): Kompetenzentwicklung in der Zeitarbeit in Frankreich Ansätze und Instrumentarium, in: Münchhausen, G. (Hrsg.): Kompetenzentwicklung in der Zeitarbeit - Potentiale und Grenzen: Bundesinstitut für Berufsbildung, Bonn

Observatoire des Métiers et de l'Emploi (2011):Trajectoire et devenir de 5000 intérimaires, November, Paris

Promberger, M. (2012): Eine Strategie oder viele Strategien? Zur Polyvalenz flexibler Beschäftigungsformen im betrieblichen Einsatz am Beispiel der Leiharbeit, in: Krause, A./Köhler, Ch. (Hrsg.): Arbeit als Ware. ZurTheorie flexibler Arbeitsmärkte, Bielefeld, S. 163-183

Schwitzer, H./Schumann, D. (2013): Tarifverträge über Branchenzuschläge eine erste Zwischenbilanz, in:WSI-Mitteilungen 66 (4), S. 297-300, http://www.boeckler.de/wsimit_2013_04_schwitzer.pdf

Schulten, Th. (2013): WSI-Mindestlohnbericht 2013 - Anhaltend schwache Min destlohnentwicklung in Europa, in: WSI-Mitteilungen 66 (2), S. 126-132,

http://www.boeckler.de/wsimit_2013_02_schulten.pdf

Siebenhüter, S. (2011): Integrationshemmnis Leiharbeit. Auswirkungen von Leiharbeit auf Menschen mit Migrationshintergrund: Otto Brenner Stiftung, Berlin

Spermann, A. (2011): The New Role of Temporary Agency Work in Germany, IZA Discussion Paper (6180), Bonn

Vanselow, A./Weinkopf, C. (2009): Zeitarbeit in europäischen Ländern - Lehren für Deutschland?: Hans-Böckler-Stiftung, Arbeitspapier (182), Düsseldorf Vermeulen, H./Oomens, S./de Wit, W./Warmerdam, J. (2012): Arbeidsmarkttransities van uitzendkrachten in de periode 2007-2010 [Labour market transitions of agency workers in the period 2007 - 2010]: ITS, Radboud University, Amsterdam 
Vermeulen, H./Warmerdam, J. (2011): Educational Monitor for the Temporary Agency Work Sector, ITS Nijmegen, October

Voss, E./Vitols, K./Farvaque, N./Broughton, A./Behling, F./Dota, F./Leonardi, S./Naedenoen, F. (2013): The Role of Temporary Agency Work and Labour MarketTransitions in Europe: Institutional frameworks, empirical evidence, good practice and the impact of social dialogue, Final Report for the Joint Eurociett/ UNI Europa Project „Temporary Agency Work and Transitions in the Labour Market", Brüsse

\section{AUTOREN}

ECKHARD VOSS, M.A., ist Politikwissenschaftler und Senior Consultant sowie Partner bei der Unternehmensberatung Wilke, Maack und Partner, Hamburg. Arbeitsschwerpunkte: Beschäftigungs- und Sozialentwicklung in Europa, vergleichende Analyse industrieller Beziehungen, sozialer Dialog und Europäisches Sozialmodell.

@ eckhard.voss@wilke-maack.de

KATRIN VITOLS, Dr. sc. pol., ist Politologin und Senior Consultant bei der Unternehmensberatung Wilke, Maack und Partner. Arbeitsschwerpunkte: Arbeits markt- und Beschäftigungssysteme, industrielle Beziehungen, Corporate Social Responsibility/Nachhaltigkeit und Corporate Governance.

katrin.vitols@wilke-maack.de 